\title{
INTEGRABLE CASES OF SATELLITE PROBLEM WITH THE THIRD BODY AND THE OBLATE PLANET
}

\author{
M. L. LIDOV
}

Institute of Mathematics, Academy of Sciences, Moscow, U.S.S.R.

\begin{abstract}
The evolutions of satellite's orbit under the influence of the third disturbing body moving on an elliptic orbit and the oblate planet are studied by averaging the disturbing function, and integrable cases are classified in seven cases.
\end{abstract}

In this report we shall study the evolution of satellite's orbit under the influence of simultaneous perturbations of the third body, moving on an elliptical orbit, and the oblateness of the planet, around which the satellite rotates.

The problem is considered in the following assumptions, formulated by Lidov (1962):

(1) The ratio of the radius-vector of the satellite $r$ and the radius-vector of the third body $r_{1}$ is small $\left(r / r_{1} \ll 1\right)$, and in the expansion of the disturbing function in the series of $r / r_{1}$ only the main term $W_{1}$ remains (Hill's approximation).

(2) The oblateness of the field, $W_{2}$, is described by the second zonal harmonic.

(3) We assume that there are no resonant relations between the frequencies of the satellite's and the third-body's rotations. In this case the disturbing function of the problem, being averaged with respect to the longitudes of the satellite and the perturbing body, $\lambda$ and $\varphi$, could be used for the description of the secular evolution:

$$
W=\frac{1}{(2 \pi)^{2}} \int_{0}^{2 \pi} \int_{0}^{2 \pi}\left(W_{1}+W_{2}\right) \mathrm{d} \lambda \mathrm{d} \varphi .
$$

In the above assumptions the following formula for $W$ is valid:

$$
\begin{aligned}
W= & \frac{\mu_{1} a^{2}}{8 a_{1}^{3}\left(1-e_{1}^{2}\right)^{3 / 2}}\left[6 e^{2}-1-15 e^{2} \sin ^{2} \omega+3 \cos ^{2} i \times\right. \\
& \left.\times\left(5 e^{2} \sin ^{2} \omega+1-e^{2}\right)\right]+\frac{\mu a_{0}^{2} c_{20}}{4 a^{3}\left(1-e^{2}\right)^{3 / 2}}\left(1-3 \cos ^{2} i_{\mathrm{eq}}\right) .
\end{aligned}
$$

Here $a, e, i, \omega$, and $\Omega$ are the conventional notations for the Kepler's elements of the satellite's orbit. The orbital plane of the perturbing body is taken as reference; $\Omega$ is counted from the ascending node of the perturbing body's orbit on the equator of the planet; $i_{\mathrm{eq}}$ is an inclination of the satellite's orbit to the equatorial plane of the planet:

$$
\cos i_{\mathrm{eq}}=\cos I \cos i-\sin I \sin i \cos \Omega,
$$

where $I$ is the angle between the orbital plane of the perturbing body and the equatorial plane, $a_{1}$ and $e_{1}$ are orbital elements of the perturbing body, $a_{0}$ is the equa- 
torial radius of the planet, $c_{20}$ is the coefficient of the second zonal harmonic, $\mu=G M$, $\mu_{1}=G M_{1}, G$ is the gravitational constant, $M$ is the mass of the central body, and $M_{1}$ is the mass of the perturbing body.

To the disturbing function (2) corresponds the system of equations (Lidov, 1962):

$$
\begin{aligned}
& \frac{\mathrm{d} a}{\mathrm{~d} n}=0, \quad \frac{\mathrm{d} \varepsilon}{\mathrm{d} n}=-(1-\varepsilon) \varepsilon^{1 / 2} \sin 2 \omega \sin ^{2} i \\
& \frac{\mathrm{d} i}{\mathrm{~d} n}=-\frac{1-\varepsilon}{2 \varepsilon^{1 / 2}} \sin 2 \omega \sin i \cos i+\beta \frac{\sin I \sin \Omega}{\varepsilon^{2}} \cos i_{\mathrm{eq}}, \\
& \frac{\mathrm{d} \omega}{\mathrm{d} n}= \varepsilon^{-1 / 2}\left[\frac{2 \varepsilon}{5}+\sin ^{2} \omega\left(\cos ^{2} i-\varepsilon\right)\right]+ \\
&+\frac{\beta}{2 \varepsilon^{2}}\left(1-5 \cos ^{2} i-\frac{2 \sin I \cos \Omega}{\sin i} \cos i_{\mathrm{eq}}\right), \\
& \frac{\mathrm{d} \Omega}{\mathrm{d} n}=-\varepsilon^{-1 / 2} \cos i\left[\frac{\varepsilon}{5}+(1-\varepsilon) \sin ^{2} \omega\right]+ \\
&+\beta\left(\varepsilon^{2} \sin i\right)^{-1}(\sin i \cos I+\cos i \sin I \cos \Omega) \cos i_{\mathrm{eq}} .
\end{aligned}
$$

Here instead of time $t$ we introduced the dimensionless time $n$ :

$$
\begin{aligned}
\mathrm{d} n & =\frac{15 \mu a^{2}}{4 a_{1}^{3} \sqrt{\mu a}\left(1-e_{1}^{2}\right)^{3 / 2}} \mathrm{~d} t, \\
\varepsilon & =1-e^{2} .
\end{aligned}
$$

$\beta$ is a constant parameter, characterizing the ratio of perturbing accelerations due to oblateness and the perturbing acceleration due to the third body,

$$
\beta=\frac{2 \mu a_{0}^{2} a_{1}^{3}\left(1-e_{1}^{2}\right)^{3 / 2} c_{20}}{5 \mu_{1} a^{5}} .
$$

Besides the obvious integral $a=$ const. the system of Equations (4) always has one more integral:

$$
W=\text { const. }
$$

where $W$ is given in (2).

The general theory says that for integrating Equations (4) the existence of one more integral is necessary. In the general case, apparently, such integral does not exist. However, in certain special cases, Equations (4) could be integrated.

Let us enumerate the following cases:

(1) For $\beta=0$ (the influence of oblateness is negligibly small) the full investigation of the problem was carried out by Lidov (1961) and Kozai (1962). In this case there exists one more integral $\varepsilon \cos ^{2} i=c_{1}$, and integral (7) could be simplified and rewritten in the following form:

$$
(1-\varepsilon)\left(\frac{2}{5}-\sin ^{2} i \sin ^{2} \omega\right)=c_{2} .
$$


To make the consideration complete we shall give, without comment, the qualitative pictures, describing the behaviour of the trajectories in the plane $(\varepsilon, \omega)$ for the three characteristic cases:

$$
\begin{array}{llll}
c_{1}>\frac{3}{5} & \text { (Figure 1a); } \quad 0<c_{1}<\frac{3}{5} \quad \text { (Figure 1b); } \\
c_{1}=0 & (\cos i=0) \quad \text { (Figure 1c). }
\end{array}
$$

(2) For $\beta= \pm \infty\left(\mu_{1}=0\right)$. The solution in this popular case was obtained by many

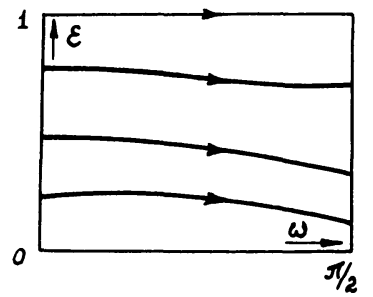

a) $c_{1}>3 / 5$

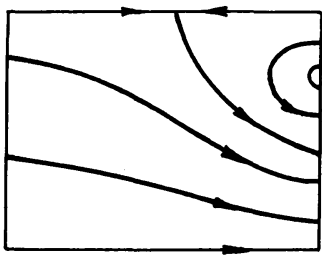

b) $0<c_{1}<3 / 5$

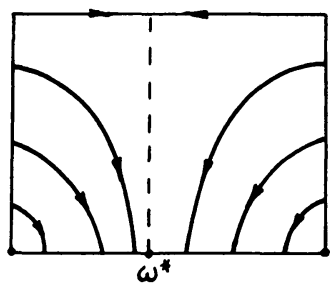

c) $c_{1}=0 \quad(\cos i=0)$

Fig. 1. $\varepsilon-\omega$ diagrams for $\beta=0$.

authors. The evolution reduces to the monotone variation of $\omega_{\mathrm{eq}}$ and $\Omega_{\mathrm{eq}}$ according to the formulas:

$$
\begin{aligned}
\frac{\mathrm{d} \omega_{\mathrm{eq}}}{\mathrm{d} t} & =\frac{\sigma}{2 \varepsilon^{2}}\left(1-5 \cos ^{2} i_{\mathrm{eq}}\right), \\
\frac{\mathrm{d} \Omega_{\mathrm{eq}}}{\mathrm{d} t} & =\frac{\sigma}{\varepsilon^{2}} \cos i_{\mathrm{eq}} .
\end{aligned}
$$

Here $\omega_{\mathrm{eq}}$ and $\Omega_{\mathrm{eq}}$ are angles counted from the equatorial plane, and,

$$
\sigma=\frac{3}{2}\left(\frac{a_{0}}{a}\right)^{2} \frac{\sqrt{\mu}}{a^{3 / 2}} c_{20}, \quad i_{\text {eq }}=\text { const. }, \quad \varepsilon=\text { const } .
$$

(3) $\sin I=0$ - the equatorial plane of the planet coincides with the orbital plane of the perturbing body.

(4) $\cos I=0, \sin i=0$ - the equatorial plane of the planet is orthogonal to the orbital plane of the perturbing body; the satellite moves in the orbital plane of the perturbing body.

(5) $\cos I=0, \cos i=0, \sin \Omega=0$ - the equatorial plane of the planet is orthogonal to the orbital plane of the perturbing body; the orbital plane of satellite coincides with the equatorial plane.

(6) $\cos i=0, \cos \Omega=0$ - the orbital plane of the satellite is orthogonal to the intersection line of the planes of the perturbing body and the equator.

(7) $\varepsilon=1(e=0)$ - circular orbits of the satellite for the arbitrary parameters $\beta, I$.

(a) In case 4 the evolution reduces to the monotone variation of the longitude of 
the pericentre of the orbit:

$$
\begin{aligned}
& \lambda=\Omega+\Delta \omega, \quad \Delta=\operatorname{sgn} \cos i \\
& \mathrm{~d} \lambda / \mathrm{d} n=\Delta\left(\varepsilon_{0}^{1 / 2} / 5+\frac{1}{2} \beta / \varepsilon^{2}\right) .
\end{aligned}
$$

Here $\varepsilon=\varepsilon_{0}=$ const., $i=i_{0}=$ const.

(b) In Figures $2 \mathrm{a}-\mathrm{d}$ the qualitative pictures of the behaviour of the trajectories for the special case, $\sin I=0$ and $\cos i=0$, are given for different values of parameter $\beta$.

The equations of the evolution in this case coincide with the equations for case 6 . That is why the qualitative pictures of the behaviour of solutions, represented in Figure 2, describe also case 6.

The equations of the evolution for case 5 also coincide with the same equations if one should replace $\beta$ by $-\frac{1}{2} \beta$ therein.

That is why one can also use the pictures in Figure 2 for the description of case 5.

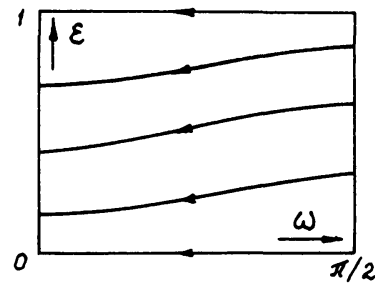

a) $\beta<-4 / 5$

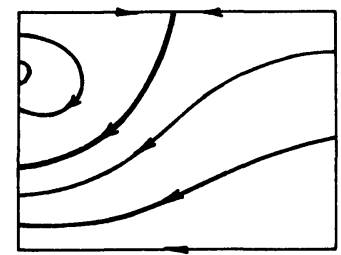

c) $0<\beta<6 / 5$

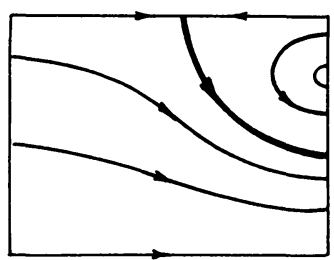

b) $-4 / 5<\beta<0$

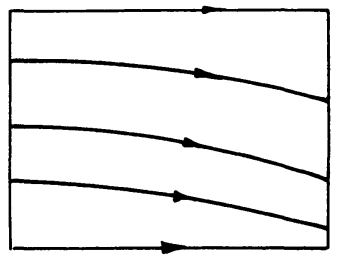

d) $\beta>6 / 5$

Fig. 2. $\varepsilon-\omega$ diagram for $\sin I=0$ and $\cos i=0$.

In this connection one should only to replace $\beta$ by $-2 \beta$ in the legends to the pictures.

Integrable case 5 was used by Lidov (1962) for the proof of stability by the eccentricity of the orbits of Uranus' satellites in spite of closeness of their inclination to the ecliptic to $90^{\circ}$.

(c) For the practically important and interesting case 7 the reader is referred to Sekiguchi (1961) and Allan and Cook (1964).

(d) The most complicated and interesting case 3 has been investigated by Kozai (1963) in connection with the problem of evolution of the orbits of the Moon's artifical satellites.

The integration of the problem in this case is based on an additional integral: the 
projection of the vector of the angular momentum on the axis of symmetry is constant. This integral may be written in the form:

$$
\varepsilon \cos ^{2} i=c_{1}=\text { const. }
$$

Using a computer Kozai (1963) constructed the field of integral curves in the plane of variables, similar to $\varepsilon, \omega$.

In our work this problem is studied completely for all the values $c_{1}$ and $\beta$. In this connection certain new cases, qualitatively different from those by Kozai, were revealed.

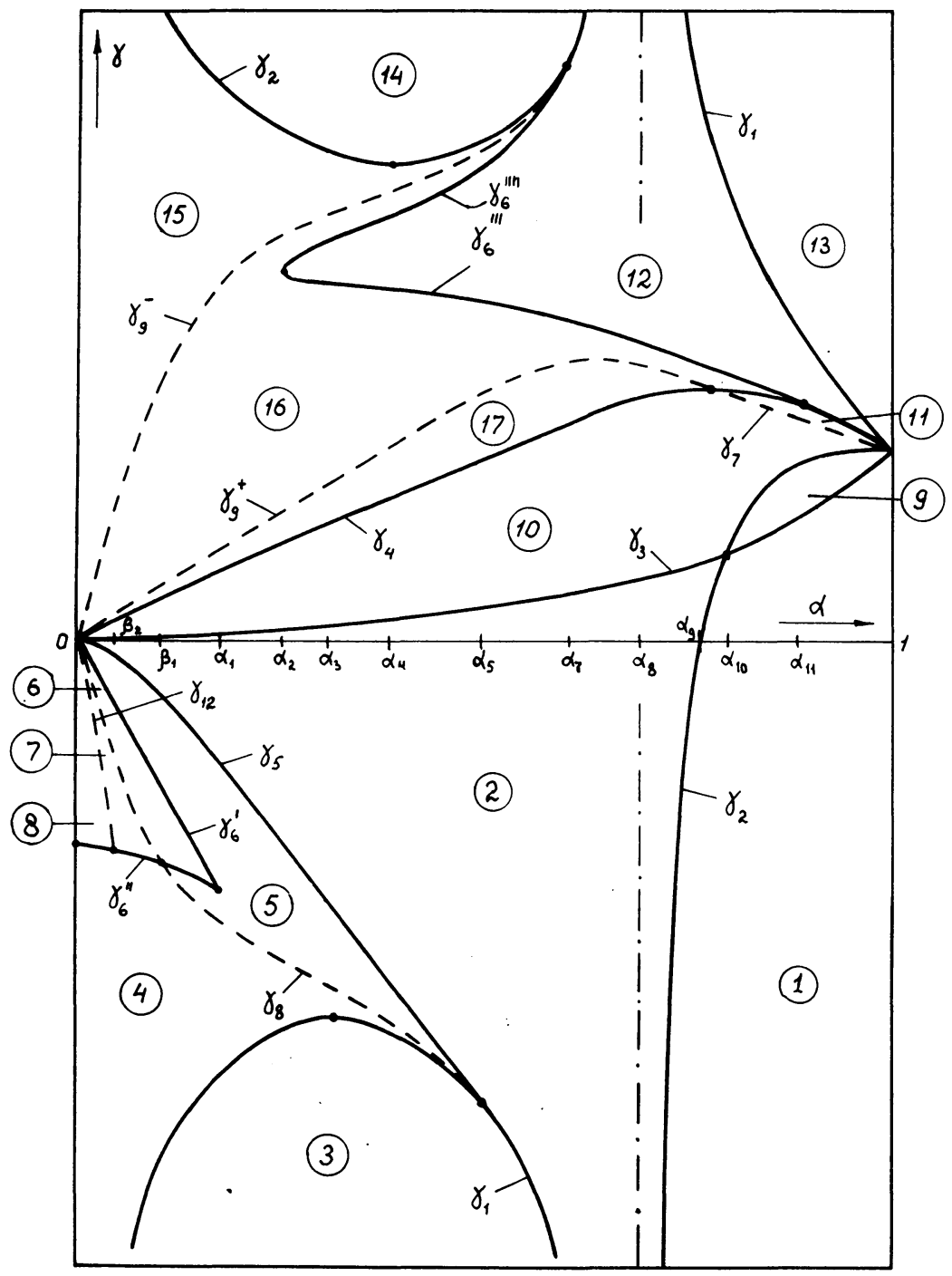

Fig. 3. Condition of appearance (or disappearance) of singularities is expressed by solid curves, and dashed curves define boundaries on which qualitative change takes place for $\sin I=0$. 

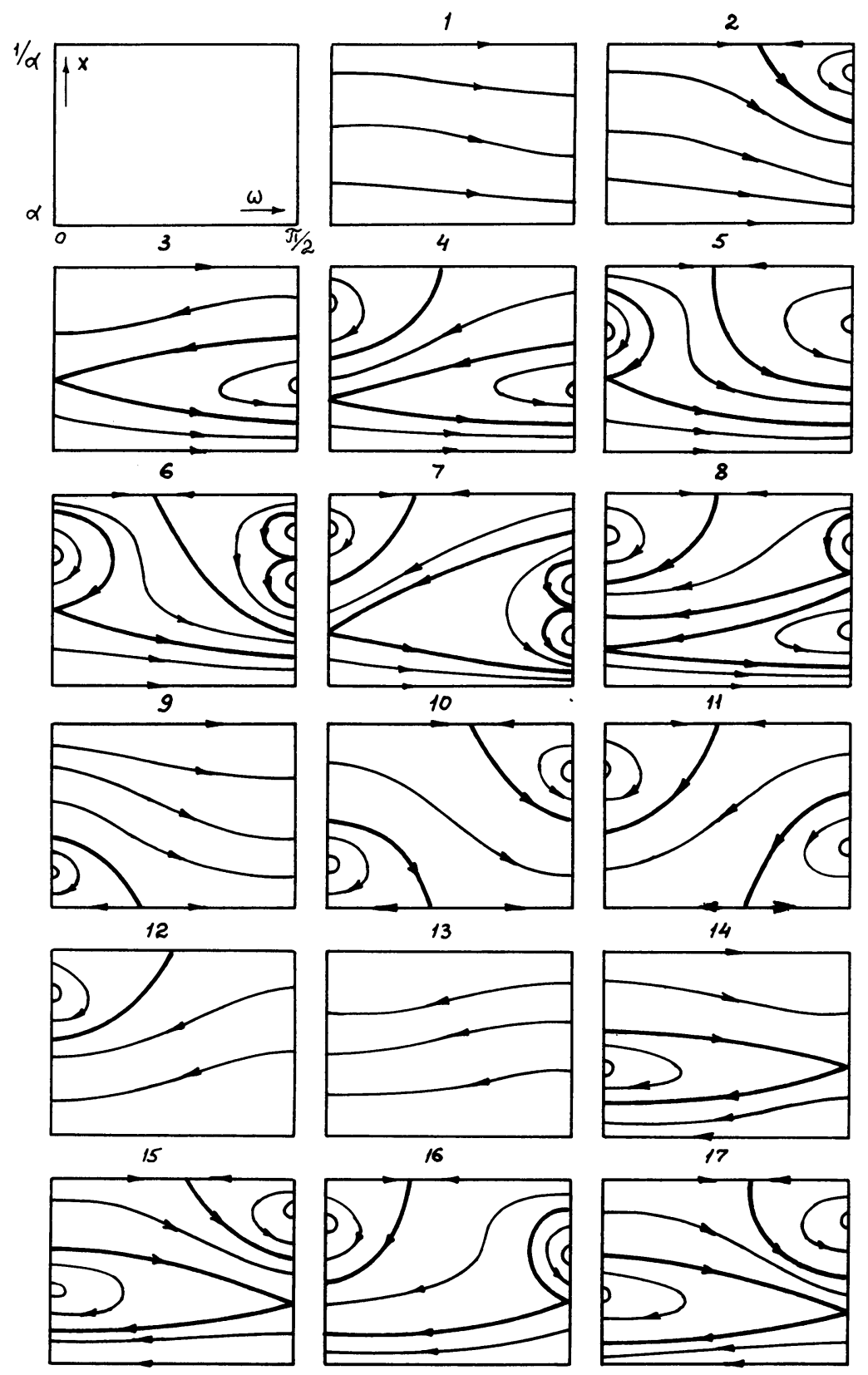

Fig. 4. $\quad \varepsilon-\omega$ diagrams for $\sin I=0$. 
In this report we shall give only certain results. A more detailed treatment is found in Lidov and Jarskaya (1973).

\section{The Investigation of Case $3(\sin I=0)$}

Instead of parameters $c_{1}$ and $\beta$ we shall use the parameters: $\gamma=\beta c_{1}^{-5 / 4}$ and $\alpha=c_{1}^{1 / 4}$. For the elliptical orbits $0 \leqslant \alpha \leqslant 1$.

Instead of variable $\varepsilon$ it turned out to be more convenient to introduce the variable $x=\varepsilon^{1 / 2} / \alpha$. It is not difficult to show that, for elliptical orbits of satellites, when $\alpha$ is fixed, $x$ could turn into the values from the interval: $\alpha \leqslant x \leqslant \alpha^{-1}$. In this investigation we studied the whole range of possible values $-\infty<\gamma<+\infty$, although in most applications we could confine ourselves to the case $\gamma \leqslant 0\left(c_{20} \leqslant 0\right)$.

In Figure 3 the strip, $0 \leqslant \alpha \leqslant 1,-\infty<\gamma<+\infty$, is divided by the solid curves $\gamma_{1}(\alpha), \gamma_{2}(\alpha), \gamma_{3}(\alpha), \gamma_{4}(\alpha), \gamma_{5}(\alpha), \gamma_{6}^{\prime}(\alpha), \ldots, \gamma_{6}^{\prime \prime \prime \prime}(\alpha)$ and by the dashed ones $\gamma_{7}(\alpha), \gamma_{8}(\alpha)$, $\gamma_{9}^{+}(\alpha), \gamma_{9}^{-}(\alpha), \gamma_{12}(\alpha)$ in 17 areas. The equations of these curves are given in Lidov and Jarskaya (1973). The construction of these curves requires a cumbersome analysis. The first group of these curves is defined by the condition of appearance (disappearance) of the singular points in the rectangle,

$$
P:\left\{\alpha \leqslant x \leqslant \alpha^{-1}, \quad 0 \leqslant \omega \leqslant \frac{1}{2} \pi\right\} .
$$

The second group of curves (the dashed ones) define the boundaries in which the qualitative change in the behaviour of separatrixrs takes place.

In Figure 4 (1-17) the qualitative pictures of the behaviour of the integral curves are given. The number of 17 pictures corresponds to the areas of parameters shown in Figure 3. In Kozai (1963) there are examples qualitatively coinciding with the cases 2, 3, 4, 5 in Figure 4.

The cases $1,6,7,8$, which also take place for $\gamma<0$ evidently, were not revealed. Let us note that the most complicated cases $6-8$ (in the rectagle $P 6$ singular points are

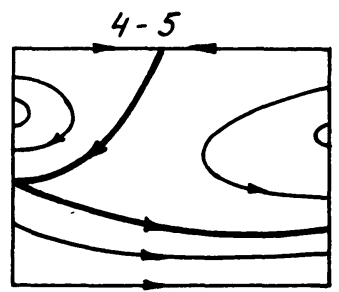

$10-11$

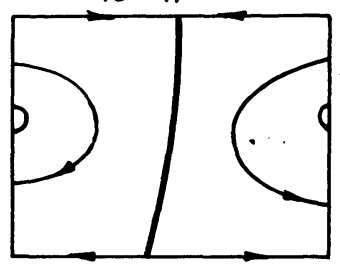

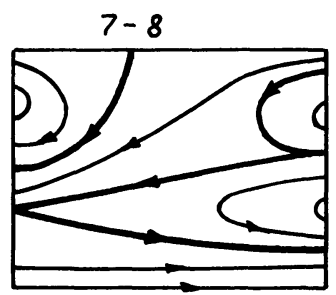

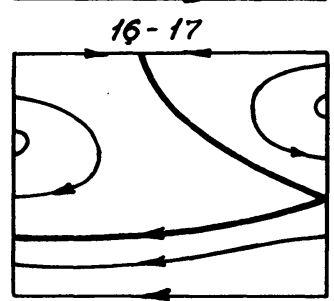

Fig. 5. $\varepsilon-\omega$ diagrams for $\sin I=0$ and for parameters of boundary values. 
present), if the eccentricity of the orbit is not too close to 1, could be realized only when the inclination of the satellite's orbit is close to $90^{\circ}$.

In Figure 5 analogous pictures are given for certain boundary values of the parameters of the problem.

For example, case 4-5 is realized if the parameters $\alpha, \gamma$ belong to the boundary, separating areas 4 and 5 represented in Figure 3.

In these cases the separatrix, going from one of the singular points of the saddle type, is at the same time the separatrix of another singular point of the same type.

The existence of many stable singular points gives reason to believe that this investigation could turn out to be useful for the practical selection of the special orbits of satellites.

\section{References}

Allan, R. R. and Cook, G. E.: 1964, Proc. Roy. Soc. A208, 97.

Kozai, Y.: 1962, Astron. J. 67, 99.

Kozai, Y.: 1963, Publ. Astron. Soc. Japan 15, 213.

Lidov, L. M.: 1961, Iskus. sputniky Zemly 8, Moscow.

Lidov, L. M.: 1962, in M. Roy (ed.), Dynamics of Satellites, Springer Verlag, Berlin, p. 168.

Lidov, L. M. and Jarskaya, M. V.: 1973, in press.

Sekiguchi, N.: 1961, Publ. Astron. Soc. Japan 19, 207. 